\title{
Con da o (meglio) con di, il corredo c'è
}

\section{Vittorio Coletti}

PUBBLICATO: 01 FEBBRAIO 2019

\begin{abstract}
Quesito:
Sono arrivate numerose (e innumerevoli circolano in rete) domande sulla reggenza di corredare, specialmente quando ha forma di participio passato o di un tempo composto con esso.
\end{abstract}

\section{Con da o (meglio) con di, il corredo c'è}

Corredato da o di?, tutti si chiedono. Speriamo di non deludere nessuno dicendo che sono ormai (purtroppo?) ammesse dall'uso entrambe le preposizioni, ma precisando che non sono uguali né ugualmente appropriate. Corredare è un verbo trivalente (cfr. la scheda Valenze e reggenze dei verbi), che produce il suo significato collegando a un soggetto due argomenti, uno diretto e uno indiretto, introdotto appunto da di: "dotare qualcosa (o qualcuno) di qualcosa (specie se utile, opportuno ecc.)". Di è la sua reggenza originaria: "Questa picciola stella si correda / d'i buoni spirti che son stati attivi", scrive Dante in Pd. VI II2-II3. E per di si possono interpretare le attestazioni registrate dall'OVI, la grande banca dati dell'italiano prequattrocentesco. Corredato di è la costruzione attestata dalle stampe cinquecentesche ed è quella degli esempi delle più antiche edizioni del Vocabolario della Crusca. Google Libri ne riporta oggi I9o.ooo attestazioni.

Dunque di, introduttore di un complemento di mezzo (anche la preposizione con si presterebbe alla bisogna, ma la lingua ha fatto da tempo, in dipendenza da corredare, la sua scelta), indispensabile per completare il significato del verbo, è la preposizione più corretta.

$\mathrm{M}$ a corredato da è oggi molto più attestato nei libri di Google (oltre un milione di volte) ed è anchesso abbastanza antico: lo si legge, sempre grazie a Google Libri, anche in un testo cinquecentesco e sono numerose le attestazioni del Settecento (il GDLI ne riporta, in mezzo a esempi tutti con di, un'unica citazione dall'Algarotti), quando, non a caso, registra la reggenza da in un esempio redazionale anche la quarta edizione del Vocabolario della Crusca.

Dunque: se di è più antica e legata alla sintassi corretta di corredare, da è anchessa assai antica e oggi molto più usata. E l'uso finisce per modificare le regole o aggiungerne di nuove, si sa.

Eppure, mentre è essenziale sapere di che cosa è corredato qualcosa o qualcuno, non è altrettanto indispensabile, ai fini del significato di corredare, sapere da chi è stata fatta questa operazione.

Ma questo da che cosa individua? Il fatto è che da ha sostituito nell'uso di, subentrando, sia pur equivocamente, nella sua funzione di introduttore di un complemento di mezzo e perdendo, o meglio riducendo quella di veicolo per l'agente o la causa efficiente.

Come si è passati da di a da?

Per rispondere esaminiamo prima la differenza originaria tra le due reggenze preposizionali. Facciamoci una domanda: se invece del participio passato corredato io usassi l'infinito corredare, userei ancora la reggenza da? No, non potrei dire: "*desidero corredare il mio libro da note". Allo stesso modo se io dico: "Giorgio ha corredato il suo libro d" belle tavole illustrate", potrei usare da? No. Ma se io uso l'infinito passivo "essere corredato" in: "spero che il mio libro possa essere corredato d* note", sento che ormai posso usare tanto di quanto da; cosi, se scrivo "Il libro di Giorgio è stato corredato d* belle tavole illustrate" al posto di $d^{*}$ mi sembra possibile tanto di quanto da. C'entrano il passivo e l'attivo? Si, e ci torneremo sopra. Intanto però dobbiamo precisare che la vera differenza tra le due preposizioni non sta tanto o solo nella diatesi del verbo che le precede, quanto nel ruolo sintattico del complemento introdotto da esse: corredato di individua un argomento del verbo che possiamo chiamare, come si diceva, complemento di mezzo, ed è indispensabile ai fini del significato del verbo stesso: "dotare, guarnire qualcosa o qualcuno di qualcosa", come abbiamo visto. Corredato da dovrebbe individuare invece un agente o una causa efficiente, tipici della frase passiva. Ma se scrivo all'attivo: "ho corredato il libro di note" e al passivo: "il libro è stato corredato di note da me" si vede che di permane anche nella frase passiva, perché introduttore sempre dello stesso argomento di mezzo, appunto il complemento richiesto da corredare, 
mentre da introduce l'agente (me, cioè io).

Ma allora perché si è imposto o si sta imponendo da?

Per più ragioni. Innanzitutto perché corredato sottintende, come tutti i participi passati di verbi transitivi, più la diatesi passiva che quella attiva e questa evoca il ruolo di agente o causa efficiente che richiededa; e poiché, spesso, l'agente è omesso, il suo introduttore, da, viene interpretato come legante verso l'essenziale (per il significato del verbo) argomento di mezzo: ecco spiegata la prevalenza odierna di da, anche se resta più corretto di. Da "il libro è stato corredato di note da me" si è passati a "il libro è stato corredato da note" con lo slittamento del mezzo alla causa agente e viceversa. Ancora un esempio: osserviamo la differenza tra "Invio il libro corredato delle mie ultime correzioni" e "Invio il libro corredato dalle ultime correzioni della redazione": usiamo il di in quella in cui il mezzo è più percepibile e il da in quella in cui si può pensare che sia l'agente o la causa efficiente. Ma nella seconda frase è davvero agente o causa efficiente il complemento introdotto conda? No; è chiaro che l'agente è la redazione o altro soggetto taciuto (un editore, ad esempio) che ne ha ripreso le note. Infatti, se volgo al passivo la frase: "la redazione ha corredato il libro di note" in "il libro è stato corredato di note dalla redazione" uso in entrambe di per individuare il complemento di mezzo e da solo in quella passiva per l'agente o causa efficiente, che è la redazione.

E tuttavia si può intravedere anche un'altra ragione, che giustifica ulteriormente la tendenza diffusa verso da. Se volgiamo al passivo una frase attiva come "belle illustrazioni corredano il libro" in "il libro è corredato da belle illustrazioni", notiamo che la funzione agente (e quindi $d a$ ) sembra proprio svolta dalle "illustrazioni", anche se è concettualmente dubbio. Ma il dubbio si può in parte superare se parafrasiamo corredare non più solo e precisamente come "dotare x di un y necessario o opportuno", ma semplicemente come, e più genericamente, "completare x", senza specificare con che cosa perché meno necessaria. Sarebbe allora in corso anche uno slittamento semantico del verbo trivalente corredare dal suo significato specifico verso quello più generico del bivalente completare, in cui il soggetto dell'attivo ("belle illustrazioni corredano il libro") può diventare al passivo un complemento d'agente con da: "Il libro è corredato (cioè completato) da belle illustrazioni". Ecco un'altra possibile ragione del successo di $d a$.

Ripetiamo: il complemento necessario a corredare, nel suo significato storico fondamentale, espresso in forma trivalente (soggetto e due argomenti, come da Sabatini-Coletti), è, oltre a quello dell'oggetto diretto, quello del complemento indiretto di mezzo introdotto da di e non quello di agente o causa efficiente introdotto con da. Ma col tempo, per la pressione del passivo, i ruoli di mezzo e d'agente (che sono del resto concettualmente meno lontani tra di loro di quanto sembri) si sono sovrapposti e da ha finito per prevalere, riassumendoli in uno solo. Per di più corredare ha diluito un po' il suo significato originario in quello più comune di "completare" bivalente, che al passivo trasforma il soggetto dell'attivo in complemento d'agente introdotto da da. Anche questa è probabilmente una ragione del crescente successo di $d a$.

Meglio di, dunque, a rigore, se si vuole rispettare il significato specifico di corredare. Ma oggi, evidentemente, l'uso percepisce da come valido sia per la materia sia per l'agente e quindi come un legante più potente, che consente oltretutto di sovrapporre al significato speciale di un verbo colto come corredare quello più comune di un verbo popolare come completare. Di qui, credo, la prevalenza vistosa di $d a$.

Dunque di o da non facciamone un dramma, anche se è un caso (non certo l'unico) in cui l'uso (con da) ha piegato la grammatica (che chiede di) e modificato la semantica. L'importante è esserne consapevoli e, nel dubbio, se vogliamo fare bella figura e adoperare il verbo corredare nel suo significato più specifico, usiamo di.

\section{Cita come:}

Vittorio Coletti, Con da o (meglio) con di, il corredo c'è , "Italiano digitale", 2019, VIII, 2019/1

(gennaio-marzo) , pp. 16-17.

DOI: $10.35948 / 2532-9006 / 2019.3064$

Copyright 2019 Accademia della Crusca

Pubblicato con licenza creative commons CC BY-NC-ND 